THE PHYSICS OF AN IGNITED TOKAMAK

\author{
F. Troyon
}

Paper presented at the 16th Symposium on Fusion Technology (SOFT) - London - 5 September 1990 


\title{
THE PHYSICS OF AN IGNITED TOKAMAK
}

\author{
F.Troyon \\ Centre de Recherches en Physique des Plasmas \\ Association Euratom - Confédération Suisse \\ Ecole Polytechnique Fédérale de Lausanne \\ 21, Av. des Bains - CH-1007 Lausanne - Switzerland
}

\section{Introduction}

There appears to be a consensus that time has come to embark on the design and construction of the next generation of tokamaks which is at the origin of the ITER initiative. Different proposals have been made based on different appreciation as to the size of the step which can be taken, related to considerations of cost, risk and duration of construction. A class of devices which may be considered the last the very high-field, high density ALCATORFrascati line of tokamaks have been proposed for some years specifically for this purpose. Today there remain three such projects: Ignitor, Ignitex and CIT. The technology chosen limits the pulse length to a few seconds. These devices have evolved through the years becoming larger and much more expensive than originally anticipated, increasing the pressure to do more than just a simple demonstration of ignition. There is another class of more ambitious devices which aim at creating long burning plasmas in conditions as close as possible to those of a tokamak reactor in order to address all the plasma physics problems associated with long burn. Three such projects, NET, the european next step after JET, ITER and JIT are good examples of this approach. The ideal would be to design a device with sufficient margin to study burning plasmas over a wide range of parameters.

The object of this didactic presentation is to describe the common physics basis of all these projects, compare their expected performance using present knowledge and list the physics problems associated with a burning plasma experiment. The comparison is not meant to be a judgement since the important parameter is the cost/benefit ratio which is a matter of appreciation at this stage. Cheaper devices can be more optimistic in their assumptions and risky. But it is nevertheless clear that if fusion research is to keep its momentum it must aim at having a device with as wide as possible a safety 
margin for ignition, address as many of the physics problems left to be solved including those specific to long burn.

\section{The DT burning parameter range}

Let us review briefly the ignition condition. A $50 \%-50 \%$ mixture of deuterium and tritium in thermal equilibrium at a temperature $T\left(T_{e}=T_{i}\right.$ measured in $\mathrm{keV}$ ) and a pressure $\mathrm{p}$ (measured in bar or $10^{5} \mathrm{~Pa}$ ) produces a fusion power

$$
P_{f}=0.075 p^{2} R(T) \quad\left(M W / m^{3}\right)
$$

where the reactivity, $R(T)$, shown in figure 1 , is normalised to unity at $T=10$ $\mathrm{keV}$.

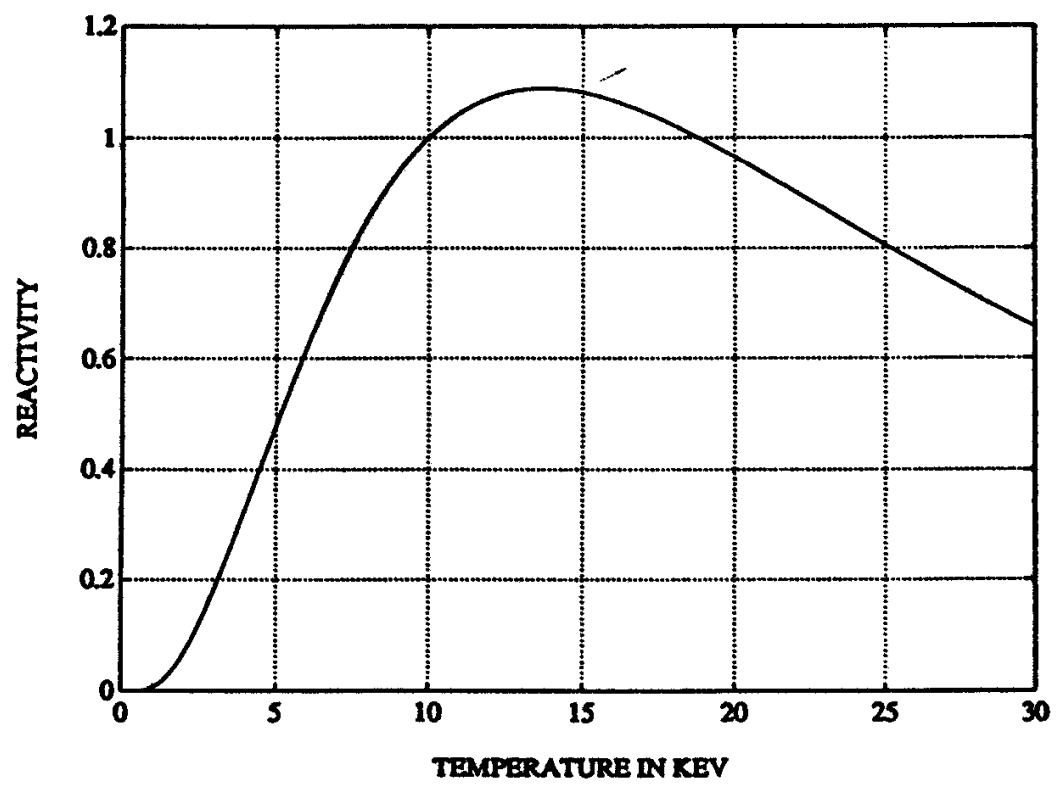

Fig.1: The reactivity of a 1:1 DT plasma, $R(T)$ as function of temperature expressed in $\mathrm{keV}$.

The power transported by the alpha-particles which can be confined and thermalised in the reacting volume to heat the bulk plasma and maintain it at the required temperature is 


$$
\mathrm{P}_{\alpha}=0.015 \mathrm{p}^{2} \mathrm{R}(\mathrm{T}) \quad\left(\mathrm{MW} / \mathrm{m}^{3}\right)
$$

Subtracting bremsstrahlung and including impurities, alpha-particles included, the net heating power available becomes

$$
\mathrm{P}_{\alpha}^{*} \approx 0.015 \mathrm{p}^{2} \mathrm{R}^{*}(\mathrm{~T}) \quad\left(\mathrm{MW} / \mathrm{m}^{3}\right)
$$

where the net reactivity is given by

$$
R^{*}(T)=\frac{4 f^{2}}{\left(1+\frac{f}{f_{D T}}\right)^{2}}\left(R(T)-\frac{10 Z_{\text {eff }}}{3 \mathrm{~T}^{2}} \mathrm{~T}^{-3 / 2}\right)
$$

with

$$
\begin{gathered}
f_{D T}=n_{D T} /\left(n_{D T}+\sum_{j} n_{j}\right), \\
f=n_{D T} / n_{e}=1-\sum_{j} n_{j} / n_{e} \text { and } \\
Z_{e f f}=1+\sum_{j} n_{j} Z_{j}\left(Z_{j}-1\right) / n_{e}
\end{gathered}
$$

and $n_{j}, n_{D T}$ and $n_{e}$ are the number densities of impurity species $j$ with ion charge $Z_{j}$, of DT ions and of electrons respectively. The plasma will be burning in quasi-steady state if the insulation of the plasma, caracterized by its cooling time, $\tau_{\mathrm{E}}$, is such that

$$
\left\langle\mathrm{p}>\tau_{\mathrm{E}}=10\left[\frac{<\mathrm{p}>^{2}}{\left\langle\mathrm{p}^{2} \mathrm{R}^{*}(\mathrm{~T})>\right.}\right], \quad\right. \text { (bar-s) }
$$

The factor in the bracket depends on the pressure and temperature profiles and on the impurity content. Figure 2 shows this condition for three illustrative cases: $5 \%$ of alpha-particles and no impurities $\left(\mathrm{Z}_{\mathrm{eff}}=1.1\right), 3 \%$ of beryllium and $5 \%$ of alpha-particles $\left(Z_{e f f}=1.45\right), 3 \%$ of carbon and $5 \%$ of alpha-particles $\left(\mathrm{Z}_{\mathrm{eff}}=2\right)$, all for flat tempature and density profiles. For reasonably peaked temperature and pressure profiles and a density weighted temperature above $10 \mathrm{keV}$ and for $\mathrm{Z}_{\text {eff }} \leq 2$ the bremsstrahlung contribution remains small and the effects of impurities and profiles factor out 


$$
<p>\tau_{E}=10 f_{\text {imp }} f_{p} \quad \text { (bar-s) }
$$

where the two factors, $f_{i m p}$ and $f_{p}$, measure respectively the effects of impurities and profiles,

$$
f_{i m p}=\frac{\left(1+\frac{f}{f D T}\right)^{2}}{4 f^{2}}, \quad f_{p} \frac{\langle p\rangle^{2}}{\left\langle p^{2} R(T)\right\rangle}
$$

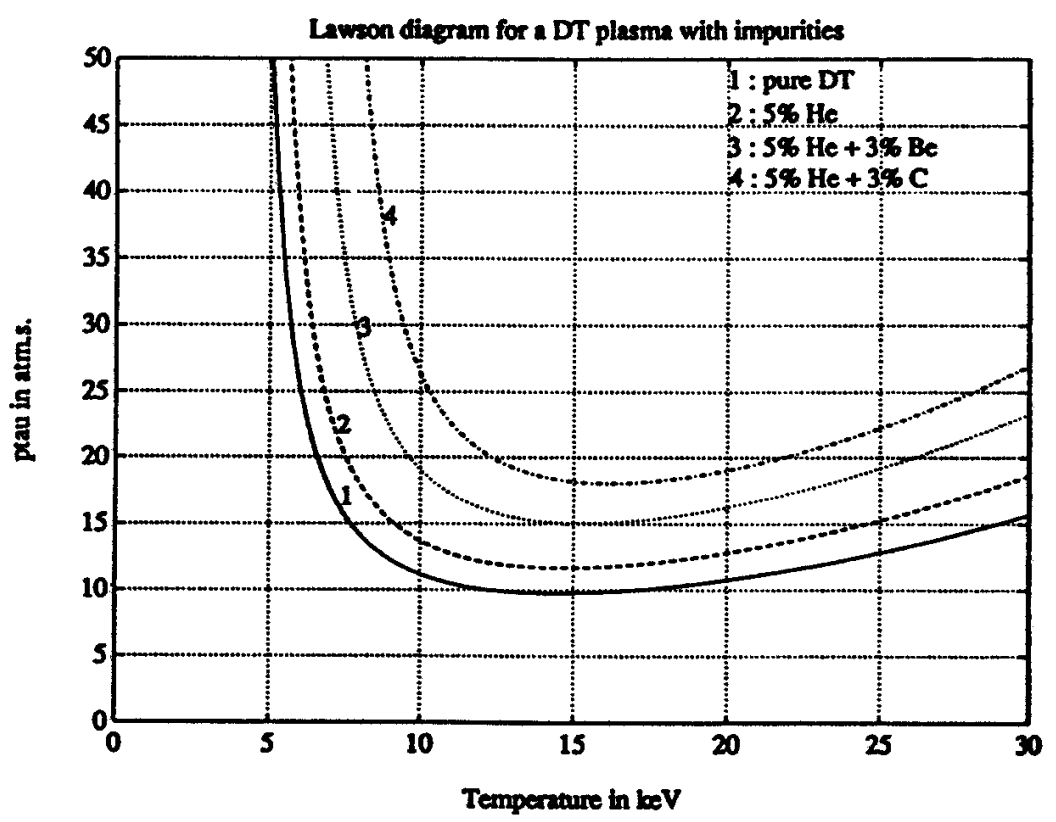

Fig.2: $\quad$ Confinement capability needed to sustain steady DT burning in plasmas having flat pressure and temperature profiles for various concentrations of impurities. The confinement capability $\langle p\rangle \tau_{E}$ is in bar.s. and the volume averaged pressure is the total plasma pressure.

Assuming that the temperature in the high pressure region is in the range 8 to $20 \mathrm{keV} \mathrm{R}(\mathrm{T})$ can be replaced by its average value 1 and

$$
f_{p}=<p>2 /<p^{2}>
$$


This factor $f_{p}$ which measures the peaking of the pressure profile varies from 0.75 for a flat density profile such as seen in $\mathrm{H}$-mode discharges when not fueled in the core and a parabolic temperature profile, to 0.45 for parabolic density and double parabolic temperature profiles. This is of the same order of magnitude as the loss in reactivity from a few percents of light impurities and alpha-particles $f_{i m p}{ }^{-1}$ as seen in fig. $1(0.85,0.65,0.56$ for the three cases). It thus seems wise to ignore both effects and consider that the gain achievable in peaking the profile will determine the level of ashes and impurities which can be accepted $\left(f_{i m p} f_{p} \approx 1\right)$. The possible gain brought by the peaking is nevertheless important in the early phase of the discharge when alpha-particles and impurities are still at a low level and the low temperature magnifies the effect of temperature peaking.

How does this steady burn condition translate into requirements on the parameters of the Tokamak?

\section{Confinement Capability of a Tokamak.}

A tokamak is defined essentially by its major radius $R$, the shape and size of the plasma cross-section usually caracterized by the small radius a, the elongation $\kappa$ and the triangularity, $\delta$, which measures the "deeness" of the shape ( $\delta=0$ is an ellipse while $\delta \approx 0.5$ for JET), the vacuum toroidal field, B, at the radius $R$ and the toroidal current , I, which circulates in the plasma. In addition the environment of the plasma is important, in particular the options of a limiter or a divertor, single null or double null.

The aspect ratio is defined as $\mathrm{R} / \mathrm{a}$ and the cylindrical safety factor $\mathrm{qI}_{\mathrm{I}}$ is defined in this paper as

$$
\mathrm{qI}=\frac{5 \mathrm{a}^{2} \mathrm{~B} \kappa}{\mathrm{IR}}
$$

To proceed further we need to relate the energy confinement time to the Tokamak parameters. In absence of a detailed theory of transport which explains all existing data it is acceptable to rely on empirical scaling laws derived from experiments and extrapolate from them. A major difficulty is the treatment of the alpha-particle heating. As evidence is increasing that plasma confinement is an intrinsic property of the plasma since different heating techniques lead to similar results, it is reasonable to assume that alpha- 
particle heating will not behave differently. It is a major objective of an ignition experiment to verify this assumption and, if possible, over as wide as possible a parameter space. Although there are a large number of such scaling laws the essential parameters are the same in most of them although there are exceptions.

We thus only consider one such scaling law to identify the trend. It is the socalled ITER-89P, developped within the ITER collaboration by P.N.Yushmanov et al.[1] and which incorporates the results of the largest devices in operation in the L-mode regime. The confinement time is given by

$$
\tau_{\mathrm{E}}=4.810^{-2} \mathrm{I}^{0.85} \mathrm{R}^{1.2} \mathrm{a}^{0.3} \mathrm{n}_{20}^{0.1} \mathrm{~B}^{0.2} \kappa^{0.5} \mathrm{P}_{\mathrm{H}^{-0.5}} \mathrm{~A}_{\mathrm{i}}^{0.5}
$$

where the current $I$ is in $\mathrm{MA}$, the heating power $\mathrm{P}_{\mathrm{H}}$ in $\mathrm{MW}, \mathrm{n}_{20}$ the electron number density in units of $10^{20} \mathrm{~m}^{-3}, \mathrm{~A}_{\mathrm{i}}$ the ion mass in proton units and the remaining quantities are in MKS units.

From the definition of the confinement time, taking care of the change of unit to bar for the pressure and approximating the plasma volume by $20 \mathrm{Rka}^{2}$

$$
<\mathrm{p}>\tau_{\mathrm{E}}=\frac{\mathrm{P}_{\mathrm{H}} \tau_{\mathrm{E}}}{3 \mathrm{R \kappa a} \mathrm{a}^{2}}
$$

Replacing $\tau_{\mathrm{E}}$ by its expression on the right-hand side of eq(2)

$$
<\mathrm{p}>\tau_{\mathrm{E}}=810^{-4} \mathrm{~A}_{\mathrm{i}} \mathrm{I}^{1.7} \mathrm{n}_{20}^{0.2} \mathrm{~B}^{0.4}\left(\frac{\mathrm{R}}{\mathrm{a}}\right)^{1.4} \quad \text { (bar. s.) }
$$

This result is independent of the heating power as for all the scaling laws which contain a confinement degradation going as $\mathrm{PH}_{\mathrm{H}}{ }^{-0.5}$, such as R.J. Goldston[2]. The exponent never seems to depart very much from this value in all scaling laws in which degradation is expressed as a power of $\mathrm{P}_{\mathrm{H}}$ as shown in the list compiled by N.A. Ucan et al.[3]. There is another class of scalings which reproduce equally well the data at high heating power, the linear offset scalings. They lead to expressions for the fusion product which increase asymptotically with the power instead of remaining constant, but in the range 
considered here they lead to the same conclusions as to the sensitivity on global parameters.

The expression (3) identifies as key parameters to increase confinement, the current and, to a lesser degree, the magnetic field. The density has a weak favorable influence, so weak one may question its reality. The favorable dependence on the aspect ratio is a bit misleading since the current is limited to a value which depends inversely on the aspect ratio.

It is better for the discussion to replace the current by the safety factor qI which caracterizes the operating range since it is limited on the low side at a value which does not depend on the magnetic field and aspect-ratio and has a weak dependence on elongation and shape.

$$
<\mathrm{p}>\tau_{\mathrm{E}}=1.210^{-2} \mathrm{~A}_{\mathrm{i}} \mathrm{qI}^{-1.7} \mathrm{\kappa}^{1.7} \mathrm{n}_{20}^{0.2} \mathrm{~B}^{2.1} \mathrm{a}^{1.7}\left(\frac{\mathrm{a}}{\mathrm{R}}\right)^{0.3} \text { (bar. s.) }
$$

The conclusion from this formula is that it is best to work at the highest magnetic field feasible, at the highest current (minimum qI ), at the lowest aspect ratio, the highest elongation and the highest density!! This shows that the performance that can be achieved will depend very much on technology limitations and by the risk one is willing to take in operating at very large elongations where data is scanty or nonexistent. The introduction of the safety factor instead of the current shows that the favorable effect of an increase of the aspect ratio suggested by the first expression is more than compensated by the necessity of reducing the current. The one obvious free parameter which is limited by cost more than by technological constraints is the size of the device.

The various proposals which have been made so far for an ignited device all start from the same assumptions: choose an elongation in the range 1.8-2.2, a value considered high but still safe, with a D-shape plasma similar to JET and DIII-D, and as low a qI as judged reasonable from present experience and theoretical predictions, in the range 1.7-2. There remains only the magnetic field and the size to play with to satisfy the ignition condition, the density having a small influence and being limited by the necessity of having the temperature sufficiently high and keeping the plasma pressure below its limit.

Table 1 shows the parameters of a few devices as they are known to me at the time of writing. The aspect ratios are around 3 and below, in the range of the tokamaks which have provided most of the data at the high elongations, large sizes and high currents nearest to the parameters of the proposed 
devices, increasing the credibility of the extrapolations. The densities quoted are not all the reference values given in the proposals as they have been adjusted to keep the opacity to the penetration of neutrals in the plasma center $\mathrm{n}_{20}$ a constant but the differences have no significant effect on the confinement capability.

The main difference between these proposals, except for JIT, is the choice of the magnet technology. The first three choose ALCATOR-like cooled magnets while the next two assume $\mathrm{NbSn}$ superconducting magnets. All five devices maximize the magnetic field and minimize the aspect ratio $\mathrm{R} / \mathrm{a}$ with their specific constraints on space, for example in NET and ITER to accomodate the neutron shield. JIT is an attempt at increasing the size to avoid working at the maximum value of the field and thus provide the possibility of scanning the field over a wide range and, if needed, leave the possibility of using another magnet technology than superconducting $\mathrm{NbSn}$ magnets.

\begin{tabular}{|l|l|l|l|l|l|l|l|l|l|}
\hline device & $\mathrm{R}$ & $\mathrm{a}$ & $\mathrm{W} / \mathrm{D}$ & $\mathrm{B}$ & $\mathrm{I}$ & $\mathrm{n}_{20}$ & $\mathrm{qI}$ & $\left\langle\mathrm{p}>\tau_{\mathrm{E}}(\mathrm{L})\right.$ & $<\mathrm{p}>\tau_{\mathrm{E}}(\mathrm{H})$ \\
\hline & & & & & & & & & \\
Ignitor & 1.20 & 0.43 & $\mathrm{~W}$ & 11 & 10 & 9 & 1.7 & $1.5(1.1)$ & $6(?)$ \\
Ignitex & 2.1 & 0.54 & $\mathrm{~W}$ & 20 & 14 & 7 & 2.0 & $5.8(5.1)$ & $22(?)$ \\
CIT & 2.1 & 0.65 & $\mathrm{D}$ & 10 & 11 & 6 & 1.8 & $2.2(3.7)$ & 10 \\
ITER & 6 & 2.15 & $\mathrm{D}$ & 4.85 & 22 & 1.8 & 1.7 & $3.5(4)$ & 15 \\
NET-II & 6.3 & 2.05 & $\mathrm{D}$ & 6 & 25 & 1.9 & 1.75 & $5.4(6.6)$ & 24 \\
JIT & 7.5 & 3 & $\mathrm{D}$ & 4.5 & 30 & 1.3 & 1.8 & $4.6(5.2)$ & 19 \\
\hline
\end{tabular}

\section{Table1}

The column $W / D$ caracterizes the edge: $W=$ contact of the plasma with the inner wall over a large area, $D=$ divertor. The density has been chosen such that the product $n_{20}$ a (the opacity to penetration of neutrals from the edge) be approximately constant. H-mode enhancement in the first two devices is doubtful because of the inner wall operation but some enhancement is postulated, in the form of transient peaked profiles with no impurities for example. The last column is the fusion product which can be expected in the $H$-mode regime assuming an enhancement factor of 2 in the confinement time.

Table 1 brings out the great similarity between these proposals with the exception of Ignitor in its present version which, if it proceeds, will undoubtly 
move to higher field, larger current or greater size. Between brackets, in the column for $\left\langle\mathrm{p}>\tau_{\mathrm{E}}\right.$ in the L-regime, are the values calculated with Goldston original scaling [2] which is always a good standard to use. Taking 10 bar.s. as the threshold of ignition, the table shows that none of the devices can reach it in the L-mode of operation. There remains a deficit which must be filled by operating in a so-called enhanced confinement regime. It should be noted that proponents of the first two devices would probably challenge the assumption that the profile effect and impurities cancel each other in very high field devices and would claim that profile effects alone are sufficient to reach ignition, a point of view which appears optimistic. The proponents of Ignitex have also proposed an upgrade of the current to $20 \mathrm{MA}$ corresponding to $\mathrm{q}_{\mathrm{I}}=1.38$ which would give a $p \tau_{E}=10$ bar.s. in L-mode operation. This low value of $q$ I is not impossible a priori because of the very low $\beta$ in this device but the validity of ITER-89P scaling for plasmas where the $q=1$ surface would be so close to the edge is doubtful. The technical feasibility of such extraordinary high values of field and current, in particular the possibility of having sufficient Volt-secs for an acceptable flat-top of the current has been questioned in technical circles. This is the reason it is not considered here.

The H-regime which is reached more easily when the tokamak operates with a divertor provides an improvement of about 2 on the confinement time and could give a satisfactory safety margin for the most performant devices as shown in the last column of the table. It should nevertheless be rembered that the gain in confinement time comes as a square in the expresion (2) for the fusion product while the gain brought by the $H$-regime is variable and is frequently less than 2. The necessity of controling impurities even at the level considered in fig.2 will most probably force such a reduction. The safety margin may thus not be as large as Table 1 suggests. Furthermore there is considerable scatter around the statistical fit provided by any scaling law which, if it is due to an inherent lack of reproducibity of tokamaks, would further reduce the safety margin.

It means there is little room left for scanning any parameter during the operation for all the devices except the last one. Any significant decrease of current, elongation, field or plasma size would rapidly reduce the prospects of a long burn if not of ignition. JIT is the only one which could increase the magnetic field and current, giving a wider range of operation and increased safety margin. This might not be acceptable because of the danger of disruptions or because of the extra cost, although it might be argued that 
because ITER is a world project one could accept paying more for a larger safety margin to achieve long burn.

The considerations above neglect the fact that ohmic heating is always present and add to the alpha-particles heating. This is justified in the ignited regime unless a new mechanism is found which enhances resisistivity. But such an enhancement would not be desirable in any long pulse device since it would imply an unbearably fast consumption of V.sec.. Ohmic heating could contribute significantly during the approach to ignition, specially in high field, short pulse devices such as Ignitor, Ignitex and CIT.

\section{The path to ignition}

The confinement capability being essentially independent of the heating power implies, loosely speaking, that auxiliary heating is only needed to bring the temperature into the range where alpha-particles heating takes over. As all scaling laws for the confinement time are statistical and do not claim to represent the evolution of a single discharge and since the transition to enhanced confinement has to occur somewhere during the approach to ignition, this is not entirely correct but the trend is certainly correct. In fig.2 the trajectory representing an igniting discharge would be a horizontal with a jump when switching to an enhanced confinement regime. The plasma pressure increases with the temperature and the pressure limit will be felt, hopefully only as an additional degradation of the confinement, thus providing a stable operating point for the burn. As long as the density has as small an influence on energy confinement as predicted by the ITER scaling law there should be no difficulty adjusting the density such that the pressure limit is above the threshold of ignition and stabilizes the burn. Figure 3 shows schematically this optimistic scenario. Plasma engineering limitations on exhaust appear today to be more constraining and may prevent working at the pressure limit, forcing an active stabilization of the burn. This would be the case certainly in very high field devices in which the thermal wall loading would be unacceptable at the pressure limit. A disruptive pressure limit would also force active stabilisation of the burn. 


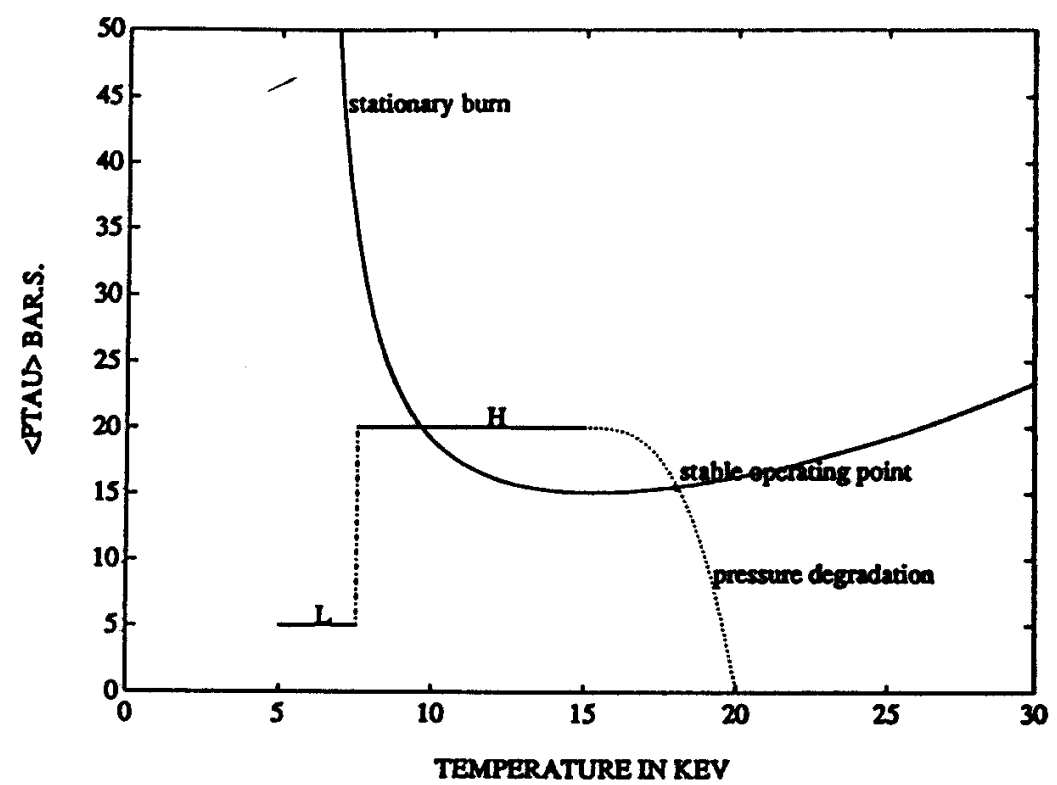

Fig.3: $\quad$ Schematic representation in the $(<p>\tau E, T)$ plane of a possible approach to a steady burn operating point $(*)$, assuming a soft degradation of the confinement near the pressure limit. The stationary burn curve is the case with $5 \% \mathrm{He}+3 \% \mathrm{Be} . L$ and $H$ refer to the $L$ and $H$-regimes respectively.

\section{Physics problems}

There are a number of problems which must be solved in addition to the fundamental requirement of having sufficient confinement capability [4]. Some can be considered as already solved or almost solved, some are serious problems that need work and some are possible roadblocks which are difficult to evaluate at this stage because of lack of experimental data and which may even turn out to be useful buttons.

\subsection{No problem...}

First in the list of physics problems essentially solved in the eighties is heating the plasma to the required temperatures. There is now a number of proven heating techniques to choose from and there is increasing evidence that enhanced confinement can be obtained with all of them. This gives freedom to 
choose the best suited system for each machine taking into account economic, technological and physics constraints, such as cost, the number of penetrations needed through the blanket and neutron shield and their area, compatibility with the parameters of the device (mainly density, magnetic field and size), minimisation of the impurity problem, possibility of some current drive assist, possibility of controling internal or edge relaxations.

From the physics point of view there no longer seems to be any convincing advantage to design a device for pure ohmic ignition, if ohmic heating degrades confinement as any other heating system. There is some preliminary evidence that enhanced confinement may also be achieved in the ohmic regime but it is not so well documented that it can be relied upon. How large is the enhancement, how wide is the parameter range in which it can be achieved and sustained, what does it require in terms of limiter design or mode of operation, are still unanswered questions. With the high cost of all devices aiming at reaching ignition and the evidence that a good enhancement is needed to have a good probability of success, it would seem wise to plan on some auxiliary heating and a confinement enhancement button such as a divertor. This is the difference between CIT which followed this line of reasoning and the other two high field proposals Ignitor and Ignitex.

The pressure limit as explained above is not an obstacle to ignition as long as the density dependence of the confinement time is as weak as in ITERP89. The observation that impurity concentration increases with decreasing density is nevertheless a reminder that it may not be easy to keep good purity if the density had to be lowered substantially below the values quoted in Table 1 . The pressure limit can be written

$$
<\mathrm{p}>_{\max }=0.04 \mathrm{gI}(\mathrm{MA}) \mathrm{B} / \mathrm{a},
$$

where $g$ depends weakly on elongation and $\mathrm{q}_{\mathrm{I}}$, and is of the order of 3 in the range of operation specified in Table 1 . With the values of densities listed in Table 1 all devices are substantially below the pressure limit at an average temperature of $10 \mathrm{keV}$, the nearest to the limit being JIT where $\langle\mathrm{p}\rangle$ would be at about $80 \%$ of the limit. For the last three devices burn controle through the pressure limit appears feasible if the limit is indeed a soft limit.

There remains a point to be clarified. At the high elongation of these devices numerical $\beta$ optimisations show that $g$ becomes a sensitive function of the current profile, a parameter which is not easy to control actively in the 
ignited regime. More experimental data in this parameter range would be useful.

Active control of the axisymmetric instability of plasmas with elongations up to 1.8 has been demonstrated in JET and up to 2.5 in DIII-D. Eliminating vertical disruptions is absolutely necessary at the level of current and field planned in these devices. It appears to be only a technological problem of providing enough peak power and sufficient frequency bandwith in the control system. The risk to be taken may be finally set by considerations of cost.

\subsection{The serious problems...}

By working at minimum aspect ratio, highest magnetic field and highest current a technological problem appears, already well-known of tokamak operators: How to obtain sufficient V.sec. in the ohmic transformer to have a long current flat top, or even just reach the desired current. There are two ways to help this problem: work at larger size, the approach followed by JIT, or use some non-inductive current drive.

The first solution can be understood by noting that the poloidal flux which must be eventually provided by the ohmic transformer scales, at constant $\mathrm{qI}$, as $\mathrm{Ba}^{2}$. At constant confinement capability $\mathrm{B}$ scales about as $\mathrm{a}^{-0.8}$ so that the poloidal flux scales as $\mathrm{a}^{1.2}$. The flux in the ohmic transformer at constant field inside the transformer scales as $\mathrm{a}^{2}$. In addition since the thickness of the shield and blanket does not increase with size, the area of the ohmic solenoid increases faster than $\mathrm{a}^{2}$. Even if stress considerations may not allow to take full benefit of the increased cross-section of the central solenoid there is a real gain, particularly important since most of the flux is consumed in the current ramp-up with a slow consumption during the flat top.

The second solution is to provide some current drive assist. During the current flat top the V.sec. consumption is low but the density is high making it impossible or at least very inefficient to use today's proven tools, lower-hybrid and medium energy neutral beams. The alternative is to assist the transformer during the start-up when density can be kept low and efficiency high. This road appears tractable but it has to be demonstrated.

The main difficulties with all present concepts for the next step and a reactor are exhaust and refueling. There are ideas to solve the exhaust problem but it is fair to say that there are still no proved solutions for burning devices. 
ITER and NET lower their reference densities from the acceptable values in Table 1 to reduce the problem of exhaust.

Exhaust has two components, energy exhaust and particle exhaust. The average energy flux which must be extracted through the scape-off, not counting the bremsstrahlung which is radiated directly to the wall, is given approximately by $0.009 \mathrm{a}<\mathrm{p}^{2}>$ in $\mathrm{MW} / \mathrm{m}^{2}$, for a 2:1 elongation. Again assuming that the gain in pressure peaking and the loss from impurities balance out this average energy flux becomes for the parameters of Table 1 about $1.2 / \mathrm{a} \mathrm{MW} / \mathrm{m}^{2}$ for an average temperature of $10 \mathrm{keV}$. For the devices in Table 1 this flux decreases monotonically from about $2.8 \mathrm{MW} / \mathrm{m}^{2}$ for an Ignitor device to about $0.4 \mathrm{MW} / \mathrm{m}^{2}$ for JIT. The corresponding neutron wall load would be about $5 / \mathrm{a}$ $\mathrm{MW} / \mathrm{m}^{2}$, about $2.5 \mathrm{MW} / \mathrm{m}^{2}$ for ITER and NET, down to about $1.6 \mathrm{MW} / \mathrm{m}^{2}$ in JIT. This is low for a reactor but it already brings enormous difficulty for the exhaust system. In a divertor the effective area which collects this heat is a small fraction of the total area, of the order of the scrape-off thickness to the radius at best giving a heat flux impossible to withstand. This is recognized as an extremely serious problem requiring much work and the extension of JET to contribute to this development is justified by the urgency of finding solutions at least for the next step. Possible solutions are to radiate as much energy as possible in the scrape-off on the way to the divertor plates, sweeping the divertor position on the target plate, thicken the scrape-off layer by some system to be found or reintroduce some form of limiter. In the first two small compact devices in Table 1 the solution proposed is to use a large fraction of the inner wall as a limiter, but even then, considering the unavoidable inhomogeneity of the energy deposition, the flux may already be excessive for some wall material. In CIT there is a divertor but a solution is not obvious considering the premium put on space in this compact device. For the next generation of devices the solution is to reduce the density. In ITER and NET the reference density is only $1.210^{20} \mathrm{~m}^{-3}$ instead of $1.910^{20} \mathrm{~m}^{-3}$, reducing the power load by a factor of about 3 and a double divertor is mentionned to gain maybe another factor of 2 .

Particle exhaust is just as demanding as energy exhaust in order to keep the level of alpha-particles sufficiently low. The relation between the ratio of particle confinement time to energy confinement time and the level of alphaparticles in the plasma during the burn is discussed in R.W. Conn [5].

Refueling the plasma with DT in the core of the plasma would be very favorable but appears impossible with known schemes except maybe with the 
smallest devices in Table 1. It is an argument made by the proponents of these compact devices that pellets could penetrate the plasma and give a chance to obtain the enhanced confinement regime associated with very peaked density profile rather than relying on the H-regime, thus making up for the lack of a divertor. This is impossible for larger devices which also cannot be fueled by neutral beams having the very high beam energy(Mev) required to penetrate such opaque plasmas. Edge fueling would probably lead to very flat density profiles and maybe favor impurity build-up in the plasma but shallow pellet injection may be sufficient for fuelling. This is still an open problem.

Impurities have plagued tokamaks since the beginning. In spite of the progress made in using low-Z materials as plasma facing materials, enhanced energy confinement regimes have almost always been associated with a strong impurity influx. With a divertor, impurities are expected to be generated at the divertor plates submitted to very severe heat and particle flows. The problem is then to minimize their production and stop them from backstreaming into the main plasma. New concepts of divertors have been offered to reach this objective but not yet demonstrated to work. But DIII-D has demonstrated recently (J.L. Luxon [6]) the possibility to controle impurities in a 10s. H-mode discharge in which every parameter is quasi-stationary with rapid small amplitude relaxations in the edge region of the plasma. The possibility of using internal relaxations of the plasma such as sawteeth and edge-mode relaxations to expell impurities has been evoked frequently but this experiment demonstrates in a spectacular manner this possibility. There remains to chart the parameter space in which this is possible, the necessary operational conditions and the associated reduction in confinement time before it can be claimed to be a proven solution for burning devices.

Disruptions have been a great source of worry because of their unpredictability and because the energy quench time does not seem to increase much with the size of the device. It means disruptions can be expected to be destructive, eroding plasma facing components and inducing large electromechanical forces in the surrounding components which may not be tolerable. A major difficulty is to predict where the current will flow in the structure and thus to compute reliably the forces. The same holds true for the toroidal and poloidal distribution of the thermal plasma energy dumped to the plasma facing components. Disruptions are usually divided into symmetric disruptions, with mainly a toroidal current induced in the structure, and vertical disruptions in which it seems that strong poloidal currents flow into 
the nearest elements of the structure and cause extremely large forces. These vertical disruptions appear to result from an insufficiently powerful position and shape control system. This does not appear insoluble although it may be very difficult at low qI and large elongation such as planned for all the devices in Table 1, and costly to provide sufficient capability to cover almost all cases. The symmetric disruptions should become more rare or slower or they would have to be controled. This is certainly one of the major problems still to be studied. The fear of disruptions has been a major element in the reluctance of the fusion community to raise the current in all ignition projects being planned even after it had become obvious that this was the only way to improve the prospects of ignition.

\subsection{Unknown territory}

The presence of a sizeable population of alpha-particles in a burning plasma may have some bad consequences on confinement according to theoretical work. On the other hand the same alpha-particles may also stabilize internal relaxations and increase pressure peaking and reactivity, which could be important in the run-up to ignition by reducing the auxiliary power required. The study of this problem is clearly one of the objectives of building ignited devices, making sure that the conditions are those expected in a reactor in order to be able to draw conclusions.

The plasma in a tokamak is never fully stationary. There are always fluctuations which can take the form of relaxations such as sawteeth in the core of the plasma and edge relaxations. These relaxations cause variations in the energy and particle flows through the plasma boundary which will further strain the exhaust system.

Creation of the plasma, elongating and shaping it, running up to ignition is a transient phase, which is important. Its cost will depend on the amount of auxiliary power needed. It has been shown in JET that in these initial phases confinement can be better than during the steady phase. The possibility of using such transient conditions, probably related to a favorable current profile, is a problem worth studying because of its impact on the cost of the device. 
One problem rarely mentionned but potentially serious for the long term future is the possibility of the discharge developping large non-axisymmetric structures which impair the quality of plasma confinement, narrow the accessible parameter range and enhance the occurence of disruptions. Although in some cases their occurence is linked to a particular mode of opertation, for example pellet injection or changes from limiter to divertor operation, they may also appear occasionnally in a particular mode of operation in one device and not in another.This reduces the reliability of tokamak operation. Certainly unacceptable in a reactor these nonaxisymmetric structures may be already unacceptable in the next step devices.

\section{Concept improvements}

Which goals could one assign to tokamak research in the near future to improve the present concepts?

Explore new exhaust systems compatible with enhanced confinement and capable to handle the energy and particle flows in a reactor is a necessity.

Extend the range of parameters studied beyond their present limits in order to estsblish if it is possible to further improve performances and give more freedom to optimize the reactor, in particular elongation and shaping since it seems to be a favorable parameter which has not been pushed to its limit because of lack of data.

Develop current drive for future use in start-up but maybe also in steady operation if the full control of the current profile opened the road to new regimes of operation with improved confinement.

The possibility that another configuration may be discovered which has better performance should not be excluded but this can only occur if speculative research is allowed to go on. There is plenty of time to do so.

\section{Conclusion}

A next step device which must demonstrate long burn and with an acceptable safety margin is possible, without speculating on the accumulation 
of favorable effects and ignoring the other ones. From the data base available today this appears possible only with large devices such as NET, ITER or JIT. If such a device is to have a sufficient range of parameters to explore, it is necessary to use the only trade-off possible today, namely to choose a conservative value of the reference magnetic field and compensate with a larger size. There remains for all devices very serious physics problems to solve, the most acute being the exhaust which must be compatible with a good quality enhanced confinement regime.

\section{$\underline{\text { References }}$}

[1] P.N. Yushmanov et al., Scalings for tokamak energy confinement, Nuclear Fusion 30 (1990) 1990-1997.

[2] R.J. Goldston, Energy confinement scaling in tokamaks. Plasma Phys. Controlled Fusion, 26 (1984) 87-104.

[3] N.A.Ucan and ITER Physics Group, ITER physics design guidelines: 1989, ITER Documentation Series, No 10, p.35, International Atomic Energy Agency, Vienna (1990).

[4] B.B. Kadomtsev, F. Troyon, M.L. Watkins and support experts, Tokamaks, Nuclear Fusion 30 (1990) 1675-1694.

[5] R.W. Conn, Boundary layer physics and implications for pumped divertors in tokamaks, Fusion Engineering and Design (1990)

[6] J.L. Luxon, G. Bramson, K.H. Burrel, et al., to appear in Plasma Phys. Controll. Fusion $32(1990)$ n 011 . 